\title{
Measurement of energy expenditure: the doubly-labelled-water method in clinical practice
}

\author{
BY W. A. COWARD \\ MRC Dunn Nutrition Unit, Downham's Lane, Milton Road, Cambridge CB4 $1 X J$
}

The doubly-labelled-water method is now being regularly exploited to measure energy expenditure in human studies. Initial differences of opinion relating to the fine details of the methodology have to a large extent been resolved, and we have arrived at a stage where it is likely that researchers unschooled in mass spectrometry and the use of stable isotopes may wish to apply the method to problems of individual interest. There is, unfortunately, a tendency for reviewers of new methods to make them sound theoretically easy and simple to apply. For example, in a well-known textbook on measurements in energy metabolism (Consolazio et al. 1963) there exists a list of what is needed to measure total body water with ${ }^{2} \mathrm{H}_{2} \mathrm{O}$ (see Table 1). Until we arrive at item 7 the equipment and materials are those that can be found in any school laboratory but at this point the need for a mass spectrometer is mentioned! In terms of cost of equipment the method changes from a matter of pence to tens of thousands of pounds and in terms of level of expertise required to something only a small number of laboratories can supply. This example perfectly illustrates the problem since the doubly-labelled-water method is little more than an extension of the measurement of total body water. Two isotopes are used rather than one, and the interest is in rates of isotopic disappearance in addition to their instantaneous dilution. Thus, the method is superficially simple but in practice something not to be embarked upon without a considerable amount of thought.

A potential user could obtain ${ }^{2} \mathrm{H}_{2} \mathrm{O}$ and $\mathrm{H}_{2}{ }^{18} \mathrm{O}$, make up an isotopic mixture according to a prescribed formula, give doses, collect samples, pass them to a commercial analytical laboratory, and then calculate results (again according to some prescribed and perhaps not very well understood formula). Some readers may view the foregoing comments as a gross exaggeration but in reality this is not the case. The understanding that stable isotopes are not radioactive is not universal among clinical scientists, mass spectrometry is frequently imagined to be as flexible a tool as the measurement of radioactive isotopes, and units of measurement (atom \%, atom \% excess, isotopic ratios and delta values relative to standards) are not widely understood. Add to this the likelihood that in clinical work many of the assumptions that exist in the doubly-labelled-water method may not simply apply, then there is considerable scope for ambiguous results to emerge from expensive and time-consuming studies.

In the ideal world one would like to see the clinical scientist entirely conversant with mass spectrometry but in practice this is probably an unreasonable demand. On the other hand we should expect a high degree of familiarity with the assumptions involved with the method. What follows is an attempt to provide some guidelines. More comprehensive information exists in a review by Coward \& Cole (1990a) and in several papers in a guide to the methodology (Prentice, 1990). The method makes several assumptions about water and carbon dioxide disappearance from the body and what happens to the ${ }^{2} \mathrm{H}$ and ${ }^{18} \mathrm{O}$ isotopes that are used to label this compartment. These are listed in Tabie 2. The usual argument that is rehearsed is that these assumptions are more-or-less correct 
Table 1. Reagents and apparatus required for body-water measurements

Reagents
1. Heavy water, deuterium oxide, $99.5 \%$
2. Dry ice
3. Ethyl alcohol (USP)
4. Acetone (CP)
5. Nitric acid (CP; used in cleaning capillary dippers)
Apparatus
1. A quantity of 20 ml ampoules
2. A gas source for sealing ampoules
3. Gas burner
4. Graduated cylinders, 250 and $1000 \mathrm{ml}$
5. Beakers, $400 \mathrm{ml}$ capacity
6. Syringe pipette, 5 ml capacity
7. A mass spectrometer

Table 2. Assumptions in the doubly-labelled-water method

1. Isotopes label only body water and carbon dioxide

2. ${ }^{2} \mathrm{H}$ is lost as water

3. ${ }^{18} \mathrm{O}$ is lost as water and $\mathrm{CO}_{2}$

4. Water and $\mathrm{CO}_{2}$ output rates are constant

5. Isotope losses are not fractionated

6. Isotope intake rates are constant

but it might be better to begin by accepting the reality that none of them is right and to ask questions about the consequences of forcing observed data on isotope disappearance into this format.

We can start by considering a set of observations as follows:

$$
\begin{aligned}
& N_{o}(\mathrm{~mol})=2451 \cdot 09(\mathrm{SE} 17 \cdot 49), \\
& N_{d}(\mathrm{~mol})=2527.81(\mathrm{SE} 14 \cdot 16), \\
& k_{o}(/ \mathrm{d})=0.10775(\mathrm{SE} 0 \cdot 00089), \\
& k_{d}(/ \mathrm{d})=-0.08282(\mathrm{SE} 0 \cdot 00070),
\end{aligned}
$$

where $N$ and $k$ are isotope dilution species and rate constants for isotope disappearance respectively. The subscripts $d$ and $o$ refer to ${ }^{2} \mathrm{H}$ and ${ }^{18} \mathrm{O}$. Since $\mathrm{CO}_{2}$ production $\left(F_{\mathrm{CO}_{2}}\right.$; $\mathrm{mol} / \mathrm{d}$ ) is measured as the difference between two outflow rates we could write:

$$
\begin{aligned}
F_{\mathrm{CO}_{2}} & =\left(N_{o} / 2\right)\left(k_{o}-k_{d}\right)=30 \cdot 55 \\
\text { or } F_{\mathrm{CO}_{2}} & =\left(N_{d} / 2\right)\left(k_{o}-k_{d}\right)=30 \cdot 51, \\
\text { or } F_{\mathrm{CO}_{2}} & =\left(k_{o} N_{o}-k_{d} N_{d}\right) / 2=27 \cdot 38 .
\end{aligned}
$$

For reasons extensively dealt with elsewhere (Prentice, 1990) it is now accepted that the last formulation is the one that should be used but a simple study of the equation shows that the relationship between $N_{o}$ and $N_{d}$ is crucial. Schoeller et al. (1986) suggested that values for $N_{o}$ and $N_{d}$ should be normalized so that $N_{d} / N_{o}$ has a value of 1.03. An average value of about this seems justified from a number of observations (Coward \& Cole, 
Table 3. Standard errors on $\mathrm{k}=0.1$ and $\mathrm{R}^{2}$ values for ten daily points on an isotope disappearance curve

\begin{tabular}{ccc}
\hline SE $(\%)$ & $R^{2}$ \\
\hline 0.5 & 0.9998 \\
1.0 & 0.9992 \\
$2 \cdot 0$ & 0.9968 \\
$3 \cdot 0$ & 0.9929 \\
4.0 & 0.9874 \\
5.0 & 0.9804 \\
\hline
\end{tabular}

$1990 a$ ), however, what is not clear, is whether the coefficient of variation observed (about $1 \%$ ) is mainly physiological or mainly analytical. If the latter is true then normalization on 1.03 is an appropriate procedure but if not then it might be preferable to use observed values. In the present context of discussing measurements in clinical practice we can say very little since we do not know how varied the ratio is in all conceivable measurement circumstances. It is certain, however, that attention should be paid to the value of the relationship and use it as a guide to the adequacy of analytical data. Values deviating markedly from 1.03 should be regarded with suspicion.

Anyone new to this field will soon discover that a further crucial point of the methodology is the measurement of $k_{o} N_{o}-k_{d} N_{d}$ differences. This difference is a relatively small value compared with those from which it is derived. This means that we need a reliable guide to the adequacy of data. Biologists are used to using $R^{2}$ values as tests of the guidelines of fit of data to a line but in this context the value is not particularly useful. Table 3 shows how the standard error on the measurement of a slope varies in relation to $R^{2}$. The aim in this methodology must be to obtain a standard error of the order of $1 \%$ of the mean but $R^{2}$ values look 'good' in a biological context for values very much larger than this.

In practice the best way to examine data is to use the guidelines suggested by Coward $\&$ Cole $(1990 a)$. If the concentration of isotope above the baseline values is divided by the amount of dose given we obtain a value $\left(C_{d}\right.$ or $\left.C_{o}\right)$ that is isotope concentration expressed relative to the dose. The reciprocal of the intercept of these values if they are plotted against time on a log scale is the zero-time isotope-dilution space.

The following relationships can also be shown:

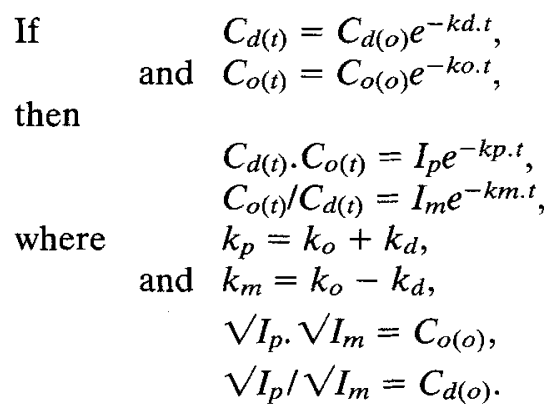

These equations form the basis of a statistical treatment of the data that allows covariance of slopes and intercepts in individual curves and the covariance of ${ }^{2} \mathrm{H}$ and ${ }^{18} \mathrm{O}$ 
disappearance to be taken into account in the calculation of errors on $\mathrm{CO}_{2}$ production rates. A full discussion of this important aspect of the work exists elsewhere (Cole et al. 1990), but for the present it will be instructive to show how appropriate plots based on these equations can be used to allow a mathematically unsophisticated worker to judge the quality of his data.

Examining plots of $\ln C_{d}$ and $\ln C_{o} v$. time for three subjects for indicating good, moderate and poor data (from Coward \& Cole, 1990a) is not particularly instructive (Fig. 1). More useful is an examination of the residuals of these plots (observed points minus fitted curves) that shows small and covariant residuals for subject 1 (Fig. 2) and large, often non-covariant residuals for subject 3 . Covariance is almost certainly a consequence of short-term variations in water output rate (this normally comprises about $80 \%$ of the total isotopic output). It is, thus, unlikely that non-covariance has a biological origin. Theoretically, it should also be expected that isotope distribution spaces calculated from early time-points should be similar to those calculated from intercepts of curves. Where residuals are large at early time-points this is not the case. Note, since ln values are used in the scales a residual of 0.01 is equivalent to a $1 \%$ difference between observed and fitted values.

Plots of the $\ln C_{o} / C_{d}$ (Fig. 3) and their residuals (Fig. 5) is a crucial test. Intercepts should have a value of +0.03 indicating $N_{d}: N_{o}$ values of 1.03 . The slope of the ratio plot is equivalent to $k_{o}-k_{d}$. Note how the data from subject 3 becomes unconvincing.

Finally, plots of $C_{o} . C_{d}$ (Fig. 4) and their residuals (Fig. 5) provide information about the constancy of water turnover. Residuals can be moderately large provided that $k_{o}-k_{d}$ indicated by the ratio plot is reasonably constant. However, biased but covariant estimates of $N_{o}$ and $N_{d}$ are indicated by large residuals at early time-points. A much more comprehensive discussion of these data is available in the work of Coward \& Cole (1990a), Cole et al. (1990) and Prentice et al. (1990). Readers wishing to pursue this topic are strongly advised to consult these articles.

The message so far ought to be clear. It is not worth worrying about fractionation corrections, background variation and isotope incorporation into non-water pools if the data with which one is working are poor. If data are less than satisfactory, questions ought to be asked about the quality of the analyses in the first place and only later about deviations away from the steady-state model.

\section{FRACTIONATION CORRECTIONS}

It is now suggested (Schoeller \& Coward, 1990) that correction for isotope losses not occurring at the same level of isotope concentrations as those that exist in body water be made on the basis of lung-water losses having a constant relationship to $\mathrm{CO}_{2}$ production and fractionated skin-water losses being dependent on body size. Values appropriate for normal individuals now exist but we do not know about the status of these in all conceivable circumstances. Given this situation it is more sensible to calculate the effects erroneous assumptions might have rather than to accept the adequacy of normal values. For example, using the relationship (Coward \& Cole, 1990a),

$$
F_{\mathrm{co}_{2}}=\frac{k_{o} N_{o}-k_{d} N_{d}-27\left(f_{2}-f_{1}\right)}{2 f_{3}+1 \cdot 1\left(f_{2}-f_{1}\right)}
$$



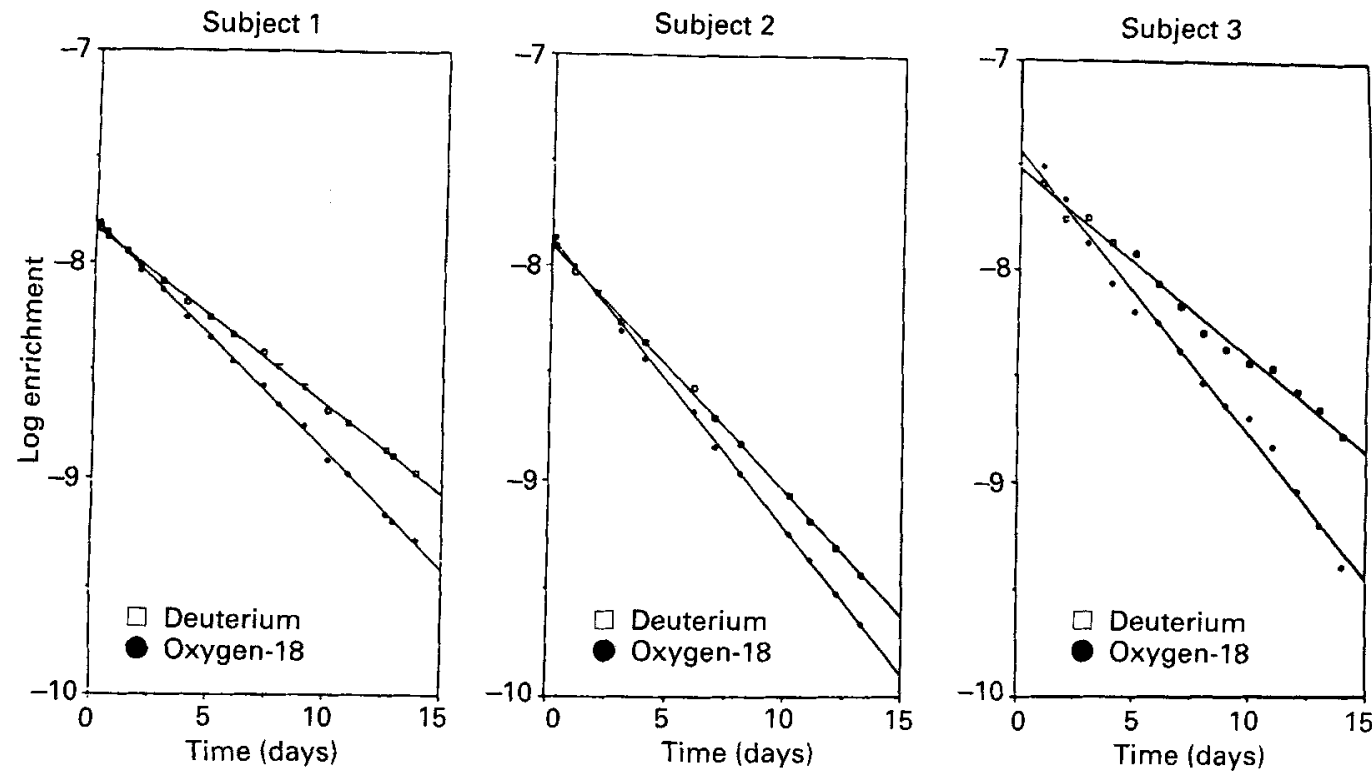

Fig. 1. In isotope concentration relative to dose for ${ }^{2} \mathrm{H}\left(C_{c t} ; \square\right)$ and ${ }^{18} \mathrm{O}\left(C_{o} ; 0\right) v$. time for three subjects undergoing measurement of energy expenditure by the doubly-labelled-water method.
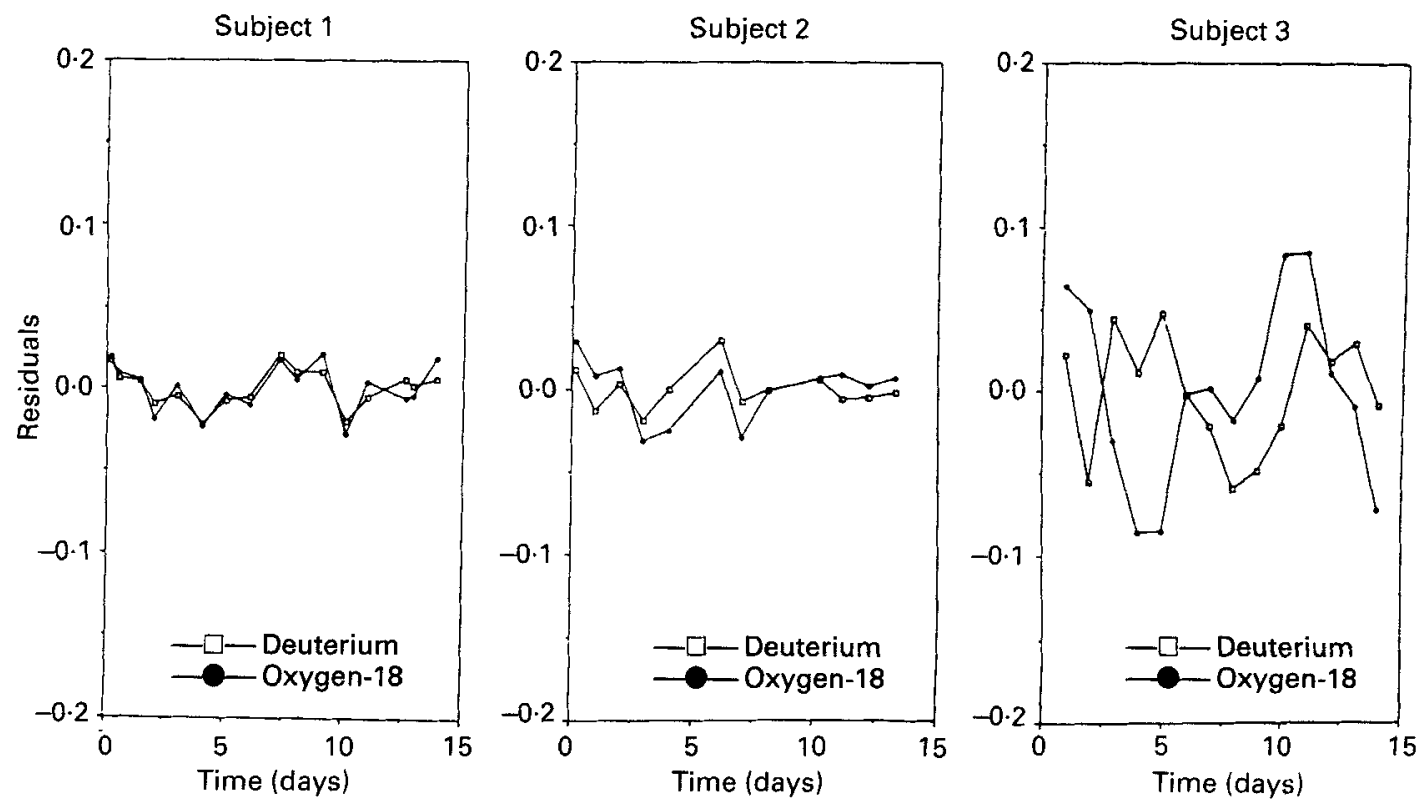

Fig. 2. Residuals of In isotope concentration relative to dose for ${ }^{2} \mathrm{H}\left(C_{d l} ; \square\right)$ and ${ }^{18} \mathrm{O}\left(C_{o} ; O\right)$ plots for three subjects undergoing measurement of energy expenditure by the doubly-labelled-water method.

where $27 \mathrm{~mol}$ water/d are fractionated as skin losses and $1.1 \mathrm{~mol}$ water $/ \mathrm{mol} \mathrm{CO}_{2}$ is fractionated as lung losses. $f_{1}, f_{2}, f_{3}$ have values of $0.941,0.992$ and 1.037 respectively and represent fractionation of ${ }^{2} \mathrm{H}$ as $\mathrm{H}_{2} \mathrm{O}\left(f_{1}\right),{ }^{18} \mathrm{O}$ as $\mathrm{H}_{2} \mathrm{O}\left(f_{2}\right)$ and ${ }^{18} \mathrm{O}$ as $\mathrm{CO}_{2}$ in equilibrium with $\mathrm{H}_{2} \mathrm{O}\left(f_{3}\right)$. Substituting the values used as an example earlier: 

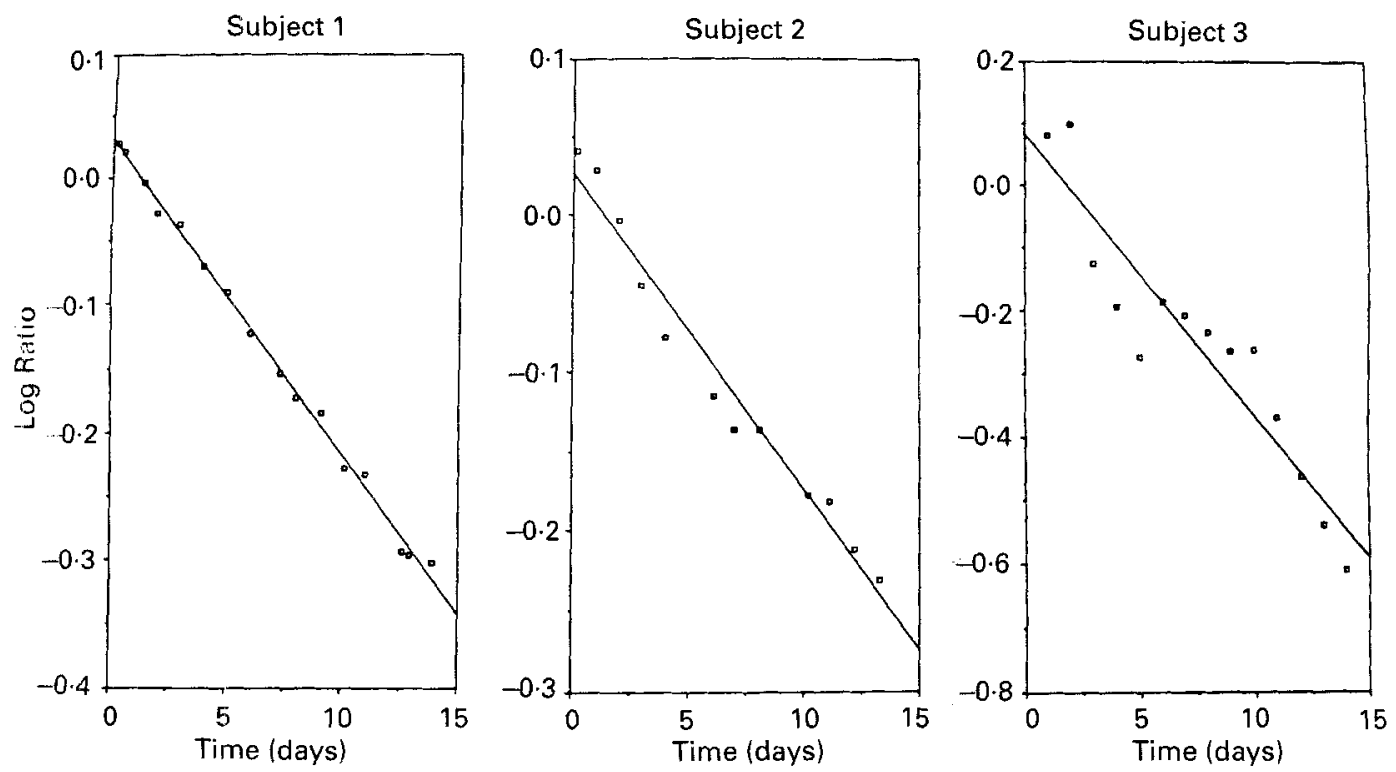

Fig. 3. In isotope concentration relative to dose for ${ }^{18} \mathrm{O}\left(C_{o}\right)$ divided by that for ${ }^{2} \mathrm{H}\left(C_{d}\right) v$. time for three subjects undergoing measurement of energy expenditure by the doubly-labelled-water method.
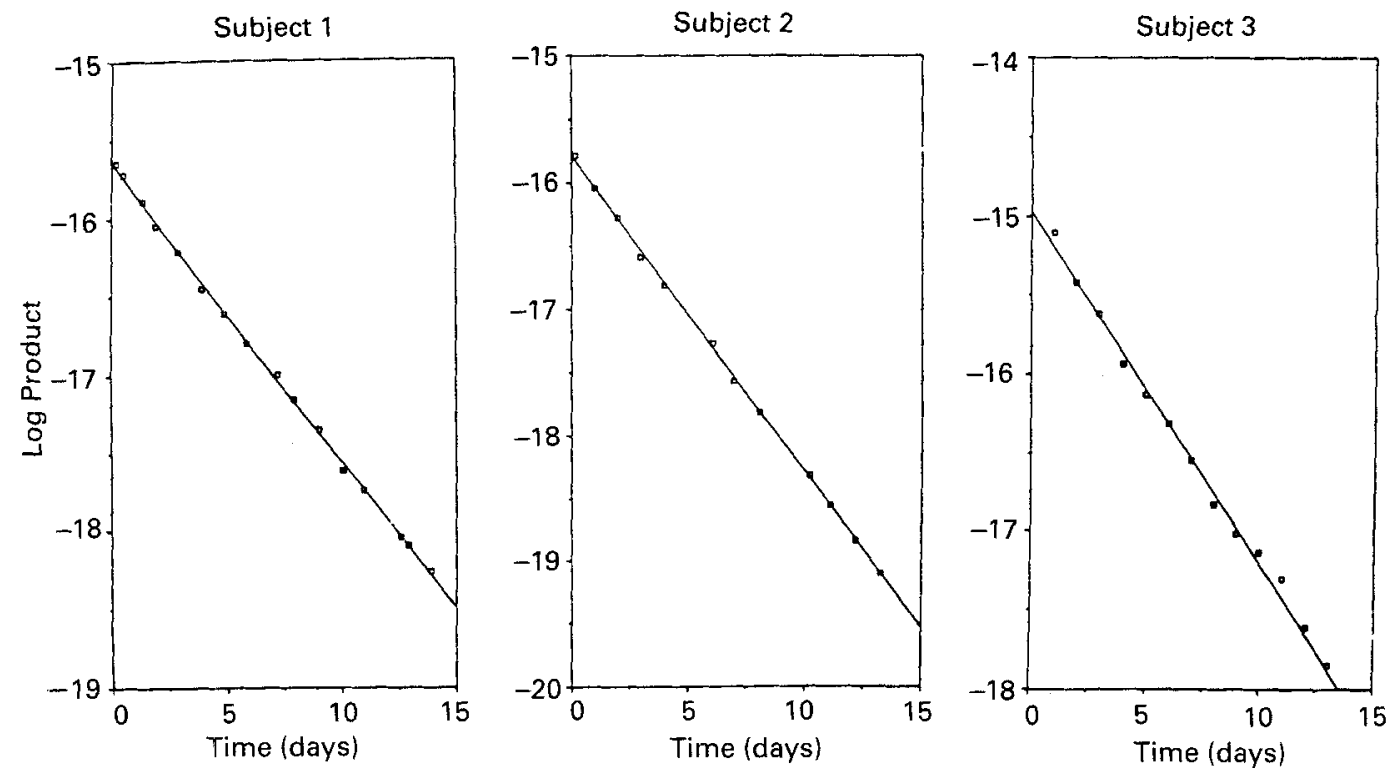

Fig. 4. In isotope concentration relative to dose for ${ }^{18} \mathrm{O}\left(C_{o}\right)$ multiplied by that for ${ }^{2} \mathrm{H}\left(C_{d}\right) v$. time for three subjects undergoing measurement of energy expenditure by the doubly-labelled-water method.

$$
F_{\mathrm{CO}_{2}}=\frac{(0.10775)(2451.09)-(0.08282)(2527 \cdot 81)-27(0.992-0.941)}{(2)(1.037)+1.1(0.992-0.941)},
$$

$F_{\mathrm{CO}_{2}}=25.057 \mathrm{~mol} / \mathrm{d}$. 

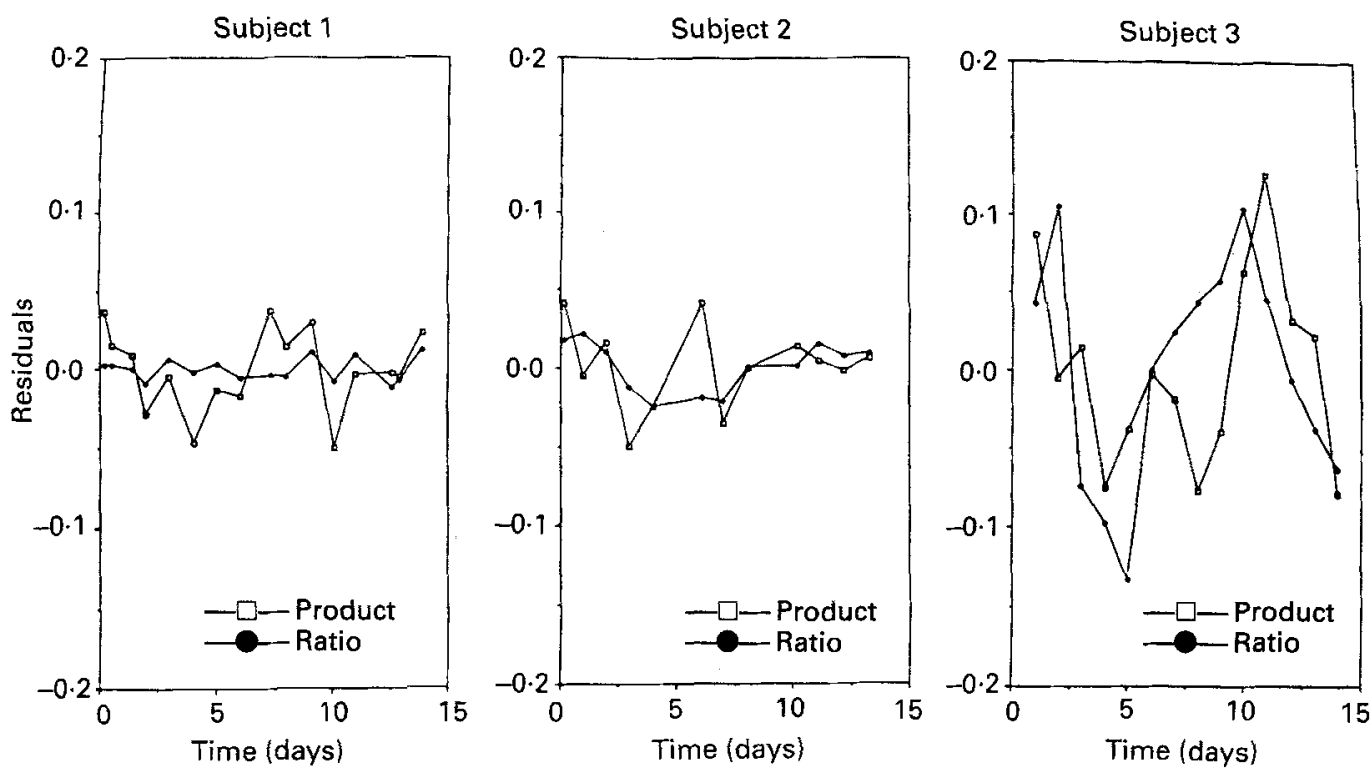

Fig. 5. Residuals of in isotope concentration relative to dose for ${ }^{18} \mathrm{O}\left(C_{o}\right)$ divided by that for ${ }^{2} \mathrm{H}\left(C_{d}\right)$ and $\ln$ $C_{o} . C_{d}$ plots for three subjects undergoing measurement of energy expenditure by the doubly-labelled-water method.

Our assumption of $27 \mathrm{~mol} / \mathrm{d}$ skin losses of fractionated water may not be correct. Imagine it should be a half or double this value. In these cases $F_{\mathrm{co}_{2}}$ calculates to be $25 \cdot 381$ or $24.411 \mathrm{~mol} / \mathrm{d}$. For this particular example the effects are relatively small but the astute reader will immediately realize that for fractionation corrections the likely magnitude of proportional errors depends on the size of the difference between $k_{o} N_{o}$ and $k_{d} N_{d}$. Furthermore the equation contains no less than five components $f_{1}, f_{2}, f_{3}$, skin fractionated water losses and the equivalence of breath water to breath $\mathrm{CO}_{2}$ whose values it is assumed are known. An error of one value in one particular direction may have only a small effect on the arithmetic but an unfortunate coincidence of a set of erroneous assumptions could have a marked effect. The reader is invited to investigate this for himself.

\section{ISOTOPE INCORPORATION INTO NON-WATER BODY-SOLIDS}

This subject has been extensively reviewed and researched by Haggarty (1990) and it seems unnecessary here to provide a comprehensive account. The main problem concerns the incorporation of ${ }^{2} \mathrm{H}$ into fat during 'de novo' fat synthesis. For the deuterium label $1 \mathrm{~g}$ fat synthesized is equivalent to $0.0296 \mathrm{~mol}$ water loss. So for calculating $\mathrm{F}_{\mathrm{CO}_{2}}$ the equation we ought to write initially is:

$$
F_{\mathrm{co}_{2}}=k_{o} N_{o}-\left(k_{d} N_{d}-0.0296 \text { Fat }\right) \text {. }
$$

In most normal circumstances (i.e. high-fat Western diets when the rate of de novo fat synthesis is small even in markedly positive energy balance) the effects of omitting this correction are likely to be small but, once again, this general statement cannot be guaranteed for all circumstances and all types of diets. It is impossible to speculate what 
the effects might be in all conceivable clinical situations, but sufficient information is provided by the simple equation to allow anyone using the method in unusual situations to define the likely magnitude of the effect.

\section{BACKGROUND VARIATION}

So far we have found that most calculated effects of inadequacies in our knowledge of what happens to ${ }^{2} \mathrm{H}$ and ${ }^{18} \mathrm{O}$ isotopes in a variety of situations are likely to be relatively small but this is not always going to be the case. The mathematics of the model used in calculations assume that the intake of ${ }^{2} \mathrm{H}$ and ${ }^{18} \mathrm{O}$ in food and water consumed by a subject during an experiment is constant. This means that the background to which isotope enrichment (following a dose) is decreasing is the same as that existing at the start of an experiment. This will not be the case if a subject is moved from his home environment and provided with food and more importantly drink or intravenous fluids of isotope composition different from that he normally consumes. In these circumstances there will be an equilibration to a new baseline. The problem, therefore, is that the background that ought to be subtracted from observed isotopic data is the final and not initial background and we may not know what this is. Again, the reader is invited to investigate for himself the likely effect of incorrect background values on $k_{o}-k_{d}$ differences. This can be done easily by calculating:

$$
k_{o}-k_{d}=\ln \frac{\left(C_{o(t 1)}-C_{o(b)}\right)}{\left(C_{d(t 1)}-C_{d(b)}\right)} \times \frac{\left(C_{d(t 2)}-C_{d(b)}\right)}{\left(C_{o(t)}-C_{o(b)}\right)} / t_{2}-t_{1},
$$

for a variety of $C_{d(b)}$ and $C_{o(b)}$ values. It will also become immediately obvious that when $C_{o(t 1)}, C_{o(t 2)}, C_{d(t 1)}$ and $C_{d(t 2)}$ values are high relative to $C_{o(b)}$ and $C_{d(b)}$ the effects are smaller than if values are closer to background and also that if $C_{o(b)}$ and $C_{d(b)}$ change in the same direction the effects are less marked than if they change in the opposite direction. Covariance of ${ }^{2} \mathrm{H}$ and ${ }^{18} \mathrm{O}$ in background is a well-defined phenomenon and occurs because fractionation effects in nature exist in the same direction for both isotopes. It follows from this that appropriate initial isotope enrichments can be calculated that will eliminate the effects of background variation if the slope of the covariance is known. Coward \& Cole $(1990 b)$ have recently described equations to calculate optimal dose levels of isotope. The relationship is surprisingly simple.

$$
\delta_{d} / \delta_{o}=\frac{S\left(2^{n}-1\right)}{\left(2^{p n}-1\right)}
$$

where $\delta_{d} / \delta_{o}$ is the ideal post-dose enrichment ratio (enrichments net of background), $S$ is the slope of covariant background changes $\left({ }^{2} \mathrm{H}\right.$ upon $\left.{ }^{18} \mathrm{O}\right), n$ is the number of biological half-lives for ${ }^{2} \mathrm{H}$ through which the experiment progresses and $p$ is $k_{o} / k_{d}$. For $S=8, p=$ 1.2 and $n=2$ a value of 5.61 emerges and is similar to the value derived by Schoeller (1983). Figs 6 and 7 show the power of using an ideal dose ratio rather than a non-ideal one when unsuspected background variation occurs.

\section{CONVERTING $\mathrm{CO}_{2}$ PRODUCTION RATES TO ENERGY EXPENDITURE VALUES}

Unfortunately, there is not a constant relationship between the amounts of $\mathrm{CO}_{2}$ and energy produced when foods are oxidized. There is a dependence on the type of food 


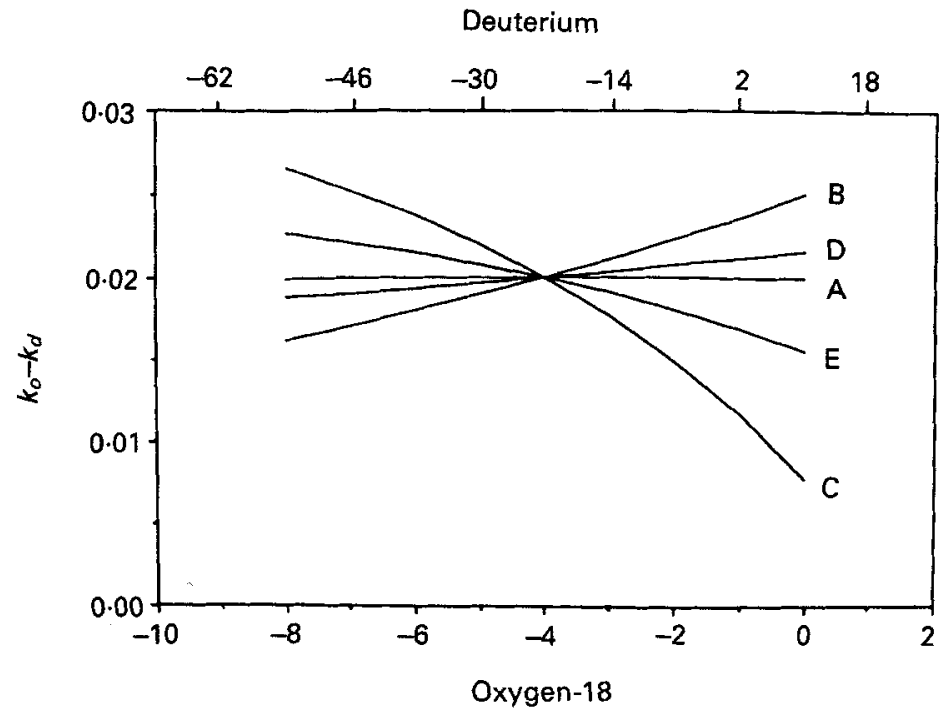

Fig. 6. Effect of background variations on estimates of rate constant for ${ }^{18} \mathrm{O}$ disappearance $\left(k_{o}\right)$ minus that for ${ }^{2} \mathrm{H}\left(k_{d}\right)$ for true $k_{o}$ and $k_{d}$ values of $0 \cdot 12$ and $0 \cdot 10$. Initial enrichment for ${ }^{18} \mathrm{O}$ rel SMOW (net of background) was $142.59 \%$ and ideal post-dose enrichment ratio $\left(\delta^{2} \mathrm{H}: \delta^{18} \mathrm{O}\right)$ values are $5 \cdot 61$ (curve A), $11 \cdot 22$ (curve $\mathrm{B}$ ), $2 \cdot 81$ (curve C), 6.68 (curve D) and 4.02 (curve E). The figure assumes covariant background changes along the meteroic water line and that the duration of the experiment was two half-lives. Correct background values were $-4\left(\delta^{18} \mathrm{O}\right)$ and $-22\left(\delta^{2} \mathrm{H}\right)$. Other positions represent background values that are incorrect.

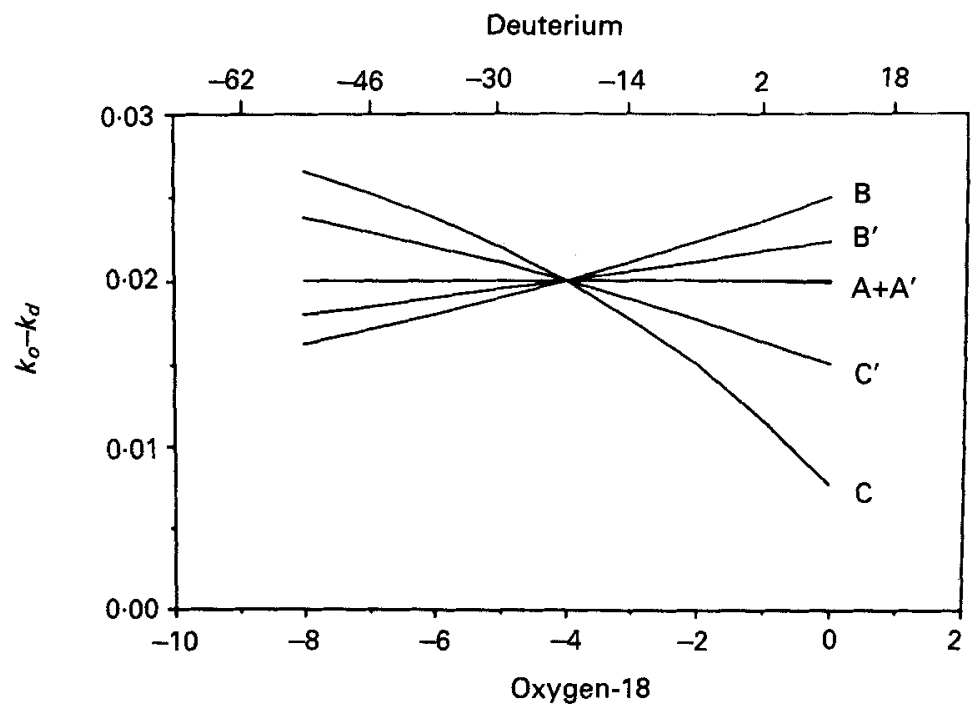

Fig. 7. Effect of background changes on estimates of rate constant for ${ }^{18} \mathrm{O}$ disappearance $\left(k_{o}\right)$ minus that for ${ }^{2} \mathrm{H}\left(k_{d}\right)$. Curves A, B, C are the same as in Fig. 6 with initial post-dose enrichment ratios $\left(\delta^{2} \mathrm{H}: \delta^{18} \mathrm{O}\right)$ of $5 \cdot 61$, $11.22,2.81,6.81$ and 4.02 respectively. A', B', C' ratios correspond to A, B, C but initial enrichments net of background are doubled. The figure assumes covariant background changes along the meteroic water line and that the duration of the experiment was two half-lives. Correct background values were $-4\left(\delta^{18} \mathrm{O}\right)$ and $-22\left(\delta^{2} \mathrm{H}\right)$. Other positions represent background values that are incorrect. 
substrate used, best described in terms of the energy equivalence of $\mathrm{CO}_{2}, E_{\mathrm{CO}_{2}}$ (Elia, 1990). Thus:

$$
E_{\mathrm{CO}_{2}}(\mathrm{~kJ} / \mathrm{l})=\frac{15 \cdot 48}{\mathrm{RQ}}+5 \cdot 55,
$$

where $\mathrm{RQ}$ is respiratory quotient, when $12 \%$ of energy expenditure is derived from protein oxidation, and the rest from a fat-carbohydrate mixture. Urinary $\mathrm{N}$ is from urea, creatinine and ammonia in the ratio 90:5:5.

Though convenient to use, this equation is a simplification of the real situation represented by the theoretically correct relationship:

$$
E_{\mathrm{CO}_{2}}=\frac{100}{p / 23 \cdot 33+f / 27 \cdot 46+c / 21 \cdot 12+a / 30 \cdot 49},
$$

where $p, f, c$ and $a$ are the percentages of total energy derived from protein, fat, carbohydrates and alcohol respectively, but since these values will in practice not be precisely known we should consider the effect of using the simpler equation. For example, an error on the $E_{\mathrm{CO}_{2}}$ of $+3 \cdot 2 \%$ would be generated if all energy was derived from protein and $-5 \cdot 8 \%$ if all energy was derived from alcohol.

For subjects in nutrient balance the $E_{\mathrm{CO}_{2}}$ that actually exists will be the same as the value that could be calculated from the mixture of foods consumed (i.e. 'RQ' of the diet is the same as true RQ, see Black et al. 1986) but there are circumstances where this will not be the case, the most common in clinical practice being the repletion of subjects receiving high-carbohydrate diets. In these circumstances $R Q$ can be greater than 1.0 and very different from that calculated from the food consumed. Calculations of fat deposition from changes in weight allow corrections to be made (Elia, 1990).

\section{CONCLUSIONS}

There is so little experience in the measurement of energy expenditure with doublylabelled water in a clinical context that it would be imprudent to suggest that investigations can be carried out without sensible 'back-up' measurements. These should include measurement of basal metabolic rate or at least resting metabolic rate, food intake and where possible body composition changes during the measurement period, the aim being to establish equivalence between energy inputs and outputs plus or minus changes in body energy stores. In other words each investigation (since each clinical circumstance investigated is probably unique) should be regarded as a partial validation of the methodology. In the event of any unexpected, ambiguous or apparently erroneous results the investigator should first check the adequacy of his analytical data and after that the adequacy of the assumptions that have been used. Experience may show that doubly-labelled water could become a useful clinical tool but we are not at the moment in a position to guarantee that this will be the case. Finally, practical considerations may prevent general utility. Clinicians usually demand that a relatively short period of time should elapse between investigations and results. To achieve this an analytical laboratory would need to be geared entirely to clinical work rather than have a more useful role in community studies. The former option would not be the best use of resources. 


\section{REFERENCES}

Black, A. E., Prentice, A. M. \& Coward, W. A. (1986). Use of food quotients to predict respiratory quotients for the doubly-labelled water method for measuring energy expenditure. Human Nutrition: Clinical Nutrition 40C, 381-391.

Cole, T. J., Franklin, M. \& Coward, W. A. (1990). Estimates of error. In The Doubly-labelled Water Method For Measuring Energy Expenditure, pp. 69-89 [A. M. Prentice, editor]. Vienna: IAEA.

Consolazio, C. F., Johnson, R. E. \& Tecora, L. J. (1963). Physiological Measurements of Metabolic Functions in Man. New York: McGraw Hill.

Coward, W. A. \& Cole, T. J. (1990a). The doubly-labelled water method for the measurement of energy expenditure in humans: risks and benefits. In New Techniques in Nutritional Research, pp. 139-176 [R. G. Whitehead, editor]. New York: Academic Press.

Coward, W. A. \& Cole, T. J. (1990b). Determination of optimum dosing ratios. In The Doubly-labelled Water Method For Measuring Energy Expenditure, pp. 294-301 [A. M. Prentice, editor]. Vienna: IAEA.

Elia, M. (1990). Converting carbon dioxide production and energy expenditure. In The Doubly-labelled Water Method For Measuring Energy Expenditure, pp. 193-211 [A. M. Prentice, editor]. Vienna: IAEA.

Haggarty, P. A. (1990). The effect of isotope sequestration and exchange. In The Doubly-labelled Water Method For Measuring Energy Expenditure, pp. 114-146 [A. M. Prentice, editor]. Vienna: IAEA.

Prentice, A. M. (1990). The Doubly-labelled Water Method For Measuring Energy Expenditure. Vienna: IAEA.

Prentice, A. M., Coward, W. A., Cole, T. J., Schoeller, D. A. \& Haggerty, P. A. (1990). In The Doubly-labelled Water Method For Measuring Energy Expenditure, pp. 212-238 [A. M. Prentice, editor]. Vienna: IAEA.

Schoeller, D. A. (1983). Energy expenditure from doubly-labelled water: some fundamental considerations in humans. American Journal of Clinical Nutrition 38, 999-1005.

Schoeller, D. A. \& Coward, W. A. (1990). Isotope fractionation connection. In The Doubly-labelled Water Method For Measuring Energy Expenditure, pp. 90-113 [A. M. Prentice, editor]. Vienna: IAEA.

Schoeller, D. A., Ravussin, E., Schutz, Y., Acheson, K. J., Baertschi, P. R. \& Jequier, E. (1986). Energy expenditure by doubly-labelled water: validation in humans and proposed calculations. American Journal of Physiology 250, R823-R830. 